\title{
Indução do estro no pós-parto em vacas primíparas Holandês-Zebu
}

[Induction of estrus in the postpartum of Holstein-Zebu heifers through norgestomet]

\author{
J.R.M. Ruas ${ }^{1,6}$, F.Z. Brandão ${ }^{2}$, J.M. Silva Filho ${ }^{3}$, A.M. Borges ${ }^{3}$, M.S. Palhares ${ }^{3}$, \\ B.C. Carvalho ${ }^{4}$, L.E. Borges 5 \\ ${ }^{1}$ Empressa de Pesquisa Agropecuária de Minas Gerais - CTZM \\ Casa 46 - Vila Gianetti \\ 36570-000 - Viçosa, MG \\ ${ }^{2}$ Departamento de Patologia e Clínica Veterinária - FVUFF - Niterói \\ ${ }^{3}$ Departamento de Clínica e Cirurgia Veterinária - EVUFMG - Belo Horizonte \\ ${ }^{4}$ Aluno de Doutorado - EVUFMG - Belo Horizonte \\ ${ }^{5}$ EPAMIG - Belo Horizonte \\ ${ }^{6}$ Pesquisador do $\mathrm{CNPq}$
}

\begin{abstract}
RESUMO
Avaliou-se o efeito do peso corporal no início do tratamento com progestágeno sobre as características reprodutivas de vacas mestiças Holandês-Zebu no pós-parto. Foram utilizadas 64 vacas, divididas em quatro grupos: GI - vacas com peso corporal entre $390-458 \mathrm{~kg}$ e submetidas a tratamento hormonal com norgestomet, GII - vacas com peso corporal entre $464-562 \mathrm{~kg}$ e submetidas a tratamento hormonal com norgestomet, GIII - vacas com peso corporal entre $374-451 \mathrm{~kg}$ (controle) e GIV - vacas com peso corporal entre $452-545 \mathrm{~kg}$ (controle). Os animais do grupo II manifestaram o primeiro estro no pós-parto mais cedo que os demais (64,4 dias - GII vs. 109,4-GI; 143,2-GIII e 105,1-GIV dias), e apresentaram menor período de serviço (94,6 dias vs. 125,5; 160,9 e 131,0 dias, na mesma ordem de citação anterior). Quanto às taxas de manifestação de estro e de gestação final, não se verificaram diferenças $(\mathrm{P}>0,05)$ entre os tratamentos. Os animais do GII apresentaram o menor período de serviço e os do GIII, o maior (94,6 vs. 160,9). Não houve influência do tratamento hormonal nem do peso corporal sobre a produção de leite e duração da lactação. O uso do implante de progestágeno nos animais que apresentaram maiores peso e condição corporal no início do tratamento respondeu por menor intervalo entre o parto e o primeiro estro. $\mathrm{O}$ uso do progestágeno em animais mais leves esteve associado ao retorno mais rápido à atividade ovariana cíclica no pós-parto.
\end{abstract}

Palavras-chave: bovino, norgestomet, pós-parto, manifestação de cio, peso corporal

\begin{abstract}
The experiment was carried out to evaluate the effect of two ranges of body weight and norgestomet treatment on the reproductive parameters of postpartum crossbred Holstein-zebu cows. Sixty four primiparous cows were randomly allocated to four treatments 40 days after calving: group I - cows with body weight ranging from 390 to $458 \mathrm{~kg}$ and norgestomet treated; group II - cows with body weight ranging from 464 to $562 \mathrm{~kg}$ and norgestomet treated; group III - cows with body weight ranging from 390 to $458 \mathrm{~kg}$ (control); and group IV - cows with body weight ranging from 464 to $562 \mathrm{~kg}$ (control). Progestagen auricular implants were mantained during 10 days and the cows were mated to bulls submitted to breeding soundness evaluation. Animals from treatment II showed estrus earlier than animals of the others treatments (II: 64.4; I: 109.4; III: 143.2 and IV: 105.1 days; $P<0.05$ ), and shorter open days (II: 94.6; I: 125.5; III: 160.9 and IV: 131.0 days; $P<0.05$ ). Estrus and pregnance rates did not
\end{abstract}

Recebido para publicação em 12 de abril de 2004

Recebido para publicação, após modificações, em 14 de outubro de 2004

E-mail: jrmruas@epamig.ufv.br 
differ between treatments $(P>0.05)$. The hormonal treatment and the body weight did not affect the total and daily milk yield, and length of lactation $(P>0.05)$. Progestagen treated, heavier and better body condition scored animals had shorter open days, and returned to postpartum ovarian ciclicity faster than lighter animals.

Keywords: cattle, norgestomet, postpartum, estrus, corporal weight

\section{INTRODUÇÃO}

Os índices de produtividade da pecuária leiteira permanecem baixos há várias décadas. Entre os fatores que contribuem para tal situação, cita-se a demora no retorno da atividade ovariana no pósparto.

A utilização dos implantes de progestágenos no pós-parto tem como objetivo inibir os neurônios produtores de $\mathrm{GnRH}$, impedindo a liberação de gonadotropinas da hipófise anterior, com maior acúmulo delas na hipófise. Ao serem retirados, ocorre liberação de grande quantidade de gonadotropinas, provocando o retorno dos ciclos ovarianos. Esses também realizam um priming de progesterona no sistema nervoso central, que impede a liberação prematura de $\mathrm{PGF}_{2 \alpha}$ por parte do endométrio, reduzindo a ocorrência de estros silenciosos e de ciclos estrais curtos. (GarciaWinder et al., 1987; Almeraya e Hidalgo, 1992; Troxel et al., 1993).

O implante com norgestomet associado ao manejo de mamadas tem sido utilizado na tentativa de melhorar os resultados de indução da ovulação e a fertilidade no pós-parto (Almeraya e Hidalgo, 1992; Oliveira Filho et al., 2000).

Belloso et al. (2002) avaliaram o uso do protocolo Crestar $^{(\mathbb{B}}$ associado ou não à remoção do bezerro em vacas primíparas mestiças (Holandês $\times$ Zebu) após 120 dias de anestro pósparto. Concluíram que a restrição de mamada ou o uso do implante de progestágeno reduziu os intervalos parto-estro e parto-primeiro serviço e aumentou a taxa de concepção em vacas mestiças.

A condição corporal da vaca ao receber o tratamento com progestágeno é um fator determinante na sua resposta ao fármaco (Almeraya e Hidalgo, 1992). Wishart et al. (1977), ao estudarem os efeitos da nutrição, peso e condição corporal sobre a fertilidade de vacas tratadas com norgestomet e valerato de estradiol, observaram maior porcentagem de fêmeas gestantes na presença de suplementação energética, calculada para se obter ganho de peso a partir de 12 semanas antes do tratamento.

Este experimento teve como objetivo verificar o efeito da hormonioterapia e do peso ao parto sobre o retorno da atividade ovariana e sobre a produção de leite por intervalo entre partos em vacas mestiças primíparas.

\section{MATERIAL E MÉTODOS}

$\mathrm{O}$ experimento foi realizado entre janeiro de 2002 e outubro de 2003. Foram utilizadas 64 vacas primíparas, mestiças $(50 \%$ Holandês $\times$ $50 \%$ Zebu), divididas ao acaso em quatro grupos experimentais, em esquema fatorial $2 \times 2$ (peso corporal $\times$ hormonioterapia), com número igual de repetições. Os grupos $(\mathrm{G})$ foram: GI - animais com peso corporal entre $390-458 \mathrm{~kg}$ e que utilizaram norgestomet $(\mathrm{n}=16)$; GII - animais com peso corporal de $464-562 \mathrm{~kg}$ e que utilizaram norgestomet $(\mathrm{n}=16)$; GIII - peso corporal entre 374-451kg; e GIV - peso corporal de $452-545 \mathrm{~kg}$.

As vacas do GI e GII receberam, em média aos 40 dias pós-parto, implante auricular ${ }^{1}$ de progestágeno $(3 \mathrm{mg}$ de norgestomet - 17 aacetoxi-11 $\beta$ - metil - 19norpregna - 4 - em 3,20diona), associado à injeção intramuscular de $3 \mathrm{mg}$ de norgestomet $+5 \mathrm{mg}$ de valerato de estradiol. O implante permaneceu durante 10 dias e, no dia de sua retirada, receberam injeção intramuscular de 333UI de gonadotrofina coriônica eqüina ${ }^{2}(\mathrm{eCG})$, seguindo o protocolo recomendado pelo fabricante. Foram, então, colocadas com touros de fertilidade comprovada.

As vacas, mantidas em pastos de capim braquiária (Brachiaria decumbens e Brachiaria

\footnotetext{
${ }^{1}$ Crestar $^{\circledR}$ - Akzo Nobel Ltda. - Divisão Intervet.

${ }^{2}$ Folligon 1000 UI - Akzo Nobel Ltda - Divisão Intervet
} 
brizantha), receberam, diariamente, ração concentrada com $22 \%$ de proteína bruta e $75 \%$ de NDT, de acordo com a produção de leite. Na época da seca, receberam silagem de milho (Zea mays, Z.) e cana-de-açúcar picada à vontade como fonte de volumoso.

A lactação foi encerrada quando, após dois controles leiteiros consecutivos, o animal produzia menos que $3 \mathrm{~kg}$ de leite diário ou quando faltavam 60 dias para o parto seguinte. As vacas foram ordenhadas duas vezes ao dia, com a presença momentânea do bezerro, sendo a primeira ordenha às $6 \mathrm{~h}$ e a segunda às $14 \mathrm{~h}$.

Foram realizados controles leiteiros a cada 28 dias e mensurações do peso e do escore corporal com a mesma freqüencia a partir do parto. A avaliação do escore corporal seguiu a escala de 1 a 5 pontos $(1=$ muito magra e $5=$ muito gorda $)$ citada por Ferreira (1990). As características reprodutivas foram avaliadas com base no dia da manifestação do estro após o parto e no número de animais gestantes após as cobrições.

Para as variáveis quantitativas (produção total de leite, duração da lactação, variação do peso corporal e retorno ao estro após o parto) aplicouse a análise de variância. Para a comparação de mais de duas médias, usou-se o teste SNK e, para a variação do escore corporal, por se tratar de uma resposta não-paramétrica, o teste KruskalWallis. As variáveis qualitativas (características reprodutivas) foram submetidas ao teste de quiquadrado (Conover, 1980; Snedecor e Cochran,
1980). Para o processamento das análises, utilizaram-se os programas SAS e o InStat 3.

O modelo estatístico usado foi:

$Y_{i j k}=M+P_{i}+N_{j}+P_{n i j}+E_{i j k}$, em que:

$Y_{\mathrm{ijk}}=$ observação na categoria de peso $\mathrm{i}$ do tratamento hormonal $\mathrm{j}$ na repetição $\mathrm{k} ; \mathrm{M}=$ efeito médio geral; $\mathrm{P}_{\mathrm{i}}=$ efeito da categoria de peso $\mathrm{i}$ ( $\mathrm{i}=$ animais leves ou pesados); $\mathrm{N}_{\mathrm{j}}=$ efeito do tratamento hormonal $\mathrm{j} \quad \mathrm{j}=$ uso ou não de norgestomet); $\mathrm{P}_{\text {nij }}=$ efeito da interação ij e $\mathrm{E}_{\mathrm{ijk}}=$ erro aleatório da observação da categoria de peso $\mathrm{i}$ no tratamento hormonal $\mathrm{j}$ na repetição $\mathrm{k}$.

\section{RESULTADOS E DISCUSSÃO}

Verificou-se homogeneidade dos grupos ao parto (Tab. 1) em relação ao peso e escore da condição corporal. Os valores de GI e GIII foram semelhantes $(\mathrm{P}>0,05)$, e o mesmo ocorreu com GII e GIV $(\mathrm{P}>0,05)$. Contudo, houve diferença quando se comparou GI e GIII com GII e GIV $(\mathrm{P}<0,05)$. Os animais perderam peso do parto até o início da aplicação do tratamento (Tab. 1), embora isso não tenha alterado a formação dos grupos experimentais, que mantiveram o mesmo padrão. Quanto ao escore da condição corporal, os animais do GIV igualaram-se aos dos grupos GI e GIII, em virtude de terem perdido mais peso que os dos demais tratamentos, com reflexo na sua condição corporal (Tab. 1).

Tabela 1. Peso médio e escore da condição corporal ao parto e quando do início e término do tratamento com implante de norgestomet (média \pm desvio-padrão)

\begin{tabular}{lcccc}
\hline \multirow{2}{*}{ Resposta } & \multicolumn{2}{c}{ Com hormonioterapia } & \multicolumn{2}{c}{ Sem hormonioterapia } \\
\cline { 2 - 5 } & Animais leves & Animais pesados & Animais leves & Animais pesados \\
\hline Peso ao parto $(\mathrm{kg})$ & $425,44 \pm 22,15 \mathrm{~b}(16)$ & $505,00 \pm 30,31 \mathrm{a}(16)$ & $425,13 \pm 21,67 \mathrm{~b}(16)$ & $494,56 \pm 30,88 \mathrm{a}(16)$ \\
Escore ao parto & $3,78 \pm 0,44 \mathrm{~b}(16)$ & $4,23 \pm 0,37 \mathrm{a}(16)$ & $3,73 \pm 0,46 \mathrm{~b}(16)$ & $4,16 \pm 0,44 \mathrm{a}(16)$ \\
Peso na aplicação do implante $(\mathrm{kg})$ & $413,63 \pm 28,83 \mathrm{~b}(16)$ & $481,56 \pm 27,61 \mathrm{a}(16)$ & $417,89 \pm 32,1 \mathrm{~b}(16)$ & $468,06 \pm 30,37 \mathrm{a}(16)$ \\
Escore na aplicação do implante & $3,39 \pm 0,43 \mathrm{~b}(16)$ & $3,87 \pm 0,58 \mathrm{a}(16)$ & $3,50 \pm 0,40 \mathrm{~b}(16)$ & $3,70 \pm 0,44 \mathrm{a}, \mathrm{b}(16)$ \\
Peso na retirada do implante $(\mathrm{kg})$ & $407,88 \pm 26,33 \mathrm{~b}(16)$ & $471,19 \pm 22,15 \mathrm{a}(16)$ & $414,38 \pm 31,51 \mathrm{~b}(16)$ & $457,50 \pm 26,01 \mathrm{a}(16)$ \\
Escore na retirada do implante & $3,38 \pm 0,39 \mathrm{~b}$ & $3,86 \pm 0,48 \mathrm{a}$ & $3,41 \pm 0,37 \mathrm{~b}$ & $3,66 \pm 0,42 \mathrm{a}, \mathrm{b}$ \\
\hline
\end{tabular}

Médias na mesma linha seguidas de letras distintas diferem $(\mathrm{P}<0,05)$ entre si pelo teste SNK. Valores entre parênteses referem-se ao $\mathrm{n}$.

A decisão de se iniciar os tratamentos com progesterona nos GI e GII aos 40 dias pós-parto, em média, partiu da premissa de que naquele momento já tivesse ocorrido a regressão morfológica e histológica do útero, isto é, já havia condições de se iniciar uma nova gestação. Além disso, presumiu-se já estar devidamente restabelecido o estoque de LH da hipófise, exaurido no final da gestação. Finalmente, considerou-se que os animais não tivessem 
perdido muito peso no período. Segundo Short et al. (1990), a involução uterina completa-se em torno de 20 a 40 dias pós-parto, não sendo a condição uterina depois desse período empecilho para o desenvolvimento de uma nova gestação. A reposição dos estoques de LH na hipófise ocorre cerca de 15 a 20 dias após a parição, o que se deve, possivelmente, ao rápido declínio da concentração plasmática, principalmente de estrógeno no periparto (Nett, 1987; Yavas e Walton, 2000).

Quanto ao intervalo entre a retirada do implante de norgestomet e a identificação do estro (Tab. 2), observa-se que ele foi menor entre os animais do grupo II, que estavam mais pesados e com maior escore da condição corporal $(\mathrm{P}<0,05)$.

Tabela 2. Retorno ao estro (dias) após a retirada do implante de norgestomet de vacas primíparas Holandês-Zebu

\begin{tabular}{lccc}
\hline Grupo experimental & $\mathrm{n}$ & Média (dias) & Desvio-padrão \\
\hline GI - 390 a 458kg com norgestomet & 16 & $69,63 \mathrm{a}$ & 85,37 \\
GII - 464 a 562kg com norgestomet & 16 & $28,00 \mathrm{~b}$ & 27,10 \\
\hline
\end{tabular}

Médias na mesma coluna seguidas de letras distintas diferem $(\mathrm{P}<0,05)$ entre si pelo teste $\mathrm{t}$.

Na Tab. 3 são mostrados os dados referentes ao retorno ao estro de todos os grupos. A hormonioterapia em vacas mais pesadas somente esteve associada à manifestação mais precoce do cio em relação às não tratadas e mais leves, isto é, o maior intervalo ocorreu nos animais do GIII e o menor, nos do GII $(\mathrm{P}<0,05)$. Os mais pesados e não tratados (GIV) responderam pelo segundo menor intervalo, seguido dos mais leves com hormonioterapia (GI). Os resultados do GI e do GII foram similares $(\mathrm{P}>0,05)$ e também não se observou diferença entre GII e GIV. Entretanto, há que se enfatizar a redução de 40,7 dias no intervalo do parto ao cio, a favor do grupo mais pesado e tratado com progesterona. Da mesma forma, com relação aos animais mais leves, deve-se ressaltar a redução de 33,8 dias no intervalo, embora esses valores não tenham sido diferentes $(\mathrm{P}>0,05)$. $\mathrm{O}$ intervalo do parto ao cio dos animais mais pesados não tratados foi apenas 4,3 dias menor do que o observado para os mais leves e tratados. Desse modo, pode-se inferir que o uso do progestágeno corrigiu, parcialmente, o efeito negativo do baixo peso ao parto sobre a característica avaliada.

Tabela 3. Retorno ao estro (dias) pós-parto de vacas primíparas Holandês-Zebu tratadas e não-tratados com implante de norgestomet (média \pm desvio-padrão)

\begin{tabular}{lcc}
\hline Peso & Tratado & Não-tratado \\
\hline Animais leves & $109,44 \pm 82,55 \mathrm{ab}(16)$ & $143,21 \pm 72,24 \mathrm{~b}(14)$ \\
Animais pesados & $64,44 \pm 26,26 \mathrm{a}(16)$ & $105,06 \pm 62,88 \mathrm{ab}(16)$ \\
\hline Médias nas mesmas linha e coluna, seguidas de letras distintas, diferem $(\mathrm{P}<0,05)$ entre si pelo teste SNK. Valores entre parênteses \\
referem-se ao $n$.
\end{tabular}

Na Tab. 4 são apresentadas as porcentagens de manifestação de estro, segundo os grupos, nos intervalos de 0-90 dias e mais de 90 dias. A taxa de manifestação de estros total durante todo o experimento não diferiu $(\mathrm{P}>0,05)$ entre os grupos. No GII, observou-se a maior manifestação de estro $(\mathrm{P}<0,05)$ nos primeiros 90 dias pós-parto, em relação aos GIII e GIV. Os animais do GI responderam pela segunda maior ocorrência de cios dentro de 90 dias pós-parto, resultado semelhante $(\mathrm{P}>0,05)$ aos do GII e GIV, porém diferente $(\mathrm{P}<0,05)$ do GIII. Dessa forma, a utilização do implante de progesterona em animais com menor peso corporal respondeu pela ocorrência semelhante de estros dentro dos primeiros 90 dias pós-parto, em relação aos animais com maior peso corporal, independentemente do uso ou não do implante de norgestomet. 
Tabela 4. Taxa de manifestação de estro (\%) em função do número de dias pós-parto em vacas primíparas mestiças Holandês-Zebu tratadas e não com implante de norgestomet

\begin{tabular}{lccc}
\hline Grupo experimental & Período pós-parto & $\mathrm{n}$ & $\%$ \\
\hline GI - 390 a 458 kg com norgestomet & $0-90$ dias & $11 / 16$ & $68,75 \mathrm{ab}$ \\
GI -390 a 458 kg com norgestomet & $>90$ dias & $5 / 16$ & 31,25 \\
Total & & $16 / 16$ & 100,00 \\
GII - 464 a 562 kg com norgestomet & $0-90$ dias & $13 / 16$ & $81,25 \mathrm{a}$ \\
GII - 464 a 562 kg com norgestomet & $>90$ dias & $3 / 16$ & 18,75 \\
Total & & $16 / 16$ & 100,00 \\
GIII - 374 a 461 kg sem norgestomet & $0-90$ dias & $5 / 16$ & $33,33 \mathrm{c}$ \\
GIII - 374 a 461 kg sem norgestomet & $>90$ dias & $10 / 16$ & 66,66 \\
Total & & $15 / 16$ & 96,25 \\
GIV - 452 a 545 kg sem norgestomet & $0-90$ dias & $7 / 16$ & $43,75 \mathrm{bc}$ \\
GIV - 452 a 545 kg sem norgestomet & $>90$ dias & $9 / 16$ & 56,25 \\
Total & & $16 / 16$ & 100,00 \\
\hline
\end{tabular}

Médias na mesma coluna seguidas de letras distintas diferem $(\mathrm{P}<0,05)$ entre si pelo teste qui-quadrado.

O comportamento no estro deve-se à alta concentração plasmática de estrógeno que, na maioria das vezes, resulta do desenvolvimento de um folículo dominante pré-ovulatório e da baixa concentração plasmática de progesterona (Driancourt, 2001). Entretanto, esse aumento de estrógeno circulante também pode ser induzido pela administração do valerato de estradiol presente nos protocolos de utilização dos implantes auriculares de norgestomet, após o término do tratamento (Larson e Kiracofe, 1995).

O desenvolvimento folicular no pós-parto, ou seja, o retorno à atividade ovariana lútea cíclica (AOLC) depende da condição e do peso corporal do animal no momento do parto e da quantidade de perda corporal no decorrer do pós-parto (Ferreira, 1991). Segundo Yavas e Walton (2000) e Sinclair et al. (2002), a condição corporal está positivamente correlacionada com a quantidade de LH na hipófise 30 dias após o parto, bem como com as concentrações circulantes de IGF-I, insulina e glicose.

Neste experimento, os animais pariram com boa condição corporal, independentemente do grupo experimental, segundo Ferreira (1991). Esse autor recomenda escore corporal no momento do parto de 3,5 a 4,0. Entretanto, 40 dias após o parto, momento em que se iniciou a hormonioterapia, observou-se que os animais perderam peso. Os mais leves estavam próximos ao limite mínimo preconizado por Ferreira (1991). Embora os animais mais pesados também tenham perdido peso, eles apresentaram maior reserva corporal, traduzida pelo maior escore da condição corporal ao parto. Assim, as perdas de peso até o início do tratamento hormonal possibilitaram que os animais mantivessem o escore corporal desejado. A condição nutricional pode interferir na função ovariana e, conseqüentemente, no retorno à AOLC no hipotálamo, na hipófise ou no ovário (Diskin et al., 2003).

Os animais que pariram com menor peso e condição corporal, ao perderem peso no pósparto, tiveram prejuízos no retorno da AOLC, traduzidos pela menor incidência de cio dentro de 90 dias pós-parto. A perda de peso no pósparto decorre basicamente da perda de tecido adiposo das reservas corporais. A mobilização delas no pós-parto é conseqüência do aumento das necessidades energéticas no início da lactação (Drackley, 1999).

O tecido adiposo é responsável pela secreção da leptina, cuja concentração circulante está altamente correlacionada com a adiposidade, que está correlacionada positivamente com as concentrações circulantes de glicose, insulina e fator de crescimento semelhante à insulina tipo 1 (IGF-1), e negativamente com as concentrações do hormônio do crescimento $(\mathrm{GH})$ e das proteínas ligadoras de IGF (IGFBD) (Vernon, et al., 2001). Embora não tenham sido quantificadas, é provável que os animais, ao perderem tecido adiposo, tenham reduzido a quantidade de gordura no pós-parto (GIII) e tenham apresentado menores concentrações circulantes de leptina, insulina, glicose e IGF-1 e maiores quantidades de IGFBP e GH.

No hipotálamo, a leptina inibe os neuropeptídeos 
orexiogênicos, como o neutropeptídeo (NPY), que atuam inibindo a secreção de GnRH. Na hipófise, a leptina estimula a síntese de LH (Ingvartesn e Boisclair, 2001). Tanto a glicose como a insulina agem no hipotálamo estimulando a síntese de GnRH. A insulina, além de atuar no hipotálamo, também o faz nos folículos, aumentando sua sensibilidade às gonadotropinas. O IGF-1 age diretamente sobre as células da teca e da granulosa, estimulando a sua proliferação e a esteroidogênese (Diskin et al., 2003). Assim, o padrão das substâncias descritas anteriormente no pós-parto terá grande importância no restabelecimento da AOLC.

O implante auricular de norgestomet, independentemente do peso corporal, foi responsável pelo retorno mais rápido ao estro pós-parto, quando se compararam os grupos tratados e não-tratados. Um dos fatores que limita o não retorno rápido à AOLC no pós-parto é a baixa concentração de LH na hipófise. O implante auricular no pós-parto tem como principal objetivo repor os estoques de $\mathrm{LH}$ hipofisários, o mais rápido possível, por meio da inibição da liberação do GnRH pelo hipotálamo. Assim, ao se retirar o implante de progestágeno, ocorre liberação de grande quantidade de gonadotropinas, acompanhada pelo retorno dos ciclos (Almeraya e Hidalgo, 1992). A ocorrência de ciclos estrais curtos pode ser evitada pelo uso do implante. Segundo Troxel et al. (1993), o norgestomet aumenta o número de animais que desenvolvem corpo lúteo de duração normal. Belloso et al. (2002) também observaram redução no intervalo até o estro na presença do implante de norgestomet.

A redução do intervalo de partos aumenta no número de partos e, conseqüentemente, aumenta a produção de leite durante a vida produtiva do animal. A hormonioterapia antecipou a manifestação do estro, refletindo em menor período de serviço (Tab. 6).

Tabela 6. Período de serviço (dias) de vacas primíparas Holandês-Zebu tratadas ou não com implante de norgestomet (média \pm desvio-padrão)

\begin{tabular}{lcc}
\hline Peso & Tratado & Não-tratado \\
\hline Animais leves & $124,46 \pm 75,39 \mathrm{ab}(14)$ & $160,93 \pm 70,57 \mathrm{a}(14)$ \\
Animais pesados & $94,63 \pm 41,31 \mathrm{~b}(16)$ & $131,00 \pm 63,93 \mathrm{ab}(16)$ \\
\hline \multicolumn{2}{l}{ Médias na mesma linha e coluna seguidas de letras distintas, diferem entre si $(\mathrm{P}<0,05)$ pelo teste SNK. Os números entre parênteses }
\end{tabular}

referem-se ao $\mathrm{n}$.

Um dos objetivos da criação de bovinos de leite é a regularidade na produção de bezerros. A AOLC está ausente no início do pós-parto, retornando apenas duas ou três semanas após o parto (Yavas e Walton, 2000). Assim, sobram apenas três ciclos para as fêmeas tornarem-se gestantes a fim de que se produza um bezerro por ano. Dessa forma, para atingir essa meta, é necessário o rápido retorno à $\mathrm{AOLC}$ no pósparto. Neste experimento, apenas os animais do GII, mais pesados e tratados com norgestomet, poderiam alcançá-la.

Barbosa Neto et al. (2001) observaram um período de gestação de 281 dias para fêmeas da raça Girolanda. Esse valor foi usado no presente experimento para determinar o intervalo de partos. A Associação Brasileira dos Criadores da Raça Girolando reconhece o período de gestação de 265 a 295 dias (média 280 dias) como aceitável para registro dos animais na associação.
A produção de leite por dia de intervalo de partos mede a associação das variáveis quantidade de leite produzido durante toda a lactação e intervalo de partos. Dessa forma, fêmeas que retornam mais precocemente à AOLC pós-parto tornam-se gestantes mais cedo. $\mathrm{Na}$ Tab. 7 apresenta-se a produção de leite por dia de intervalo de partos. $\mathrm{O}$ maior peso corporal dos animais associado ao uso do implante de norgestomet não resultou no aumento da produção de leite por dia de intervalo de partos $(\mathrm{P}>0,05)$. Neste experimento, a lactação foi encerrada quando faltavam 60 dias para o próximo parto ou quando a produção diária era inferior a $3 \mathrm{~kg}$, após dois controles leiteiros consecutivos. Assim, a produção total de leite foi influenciada pela duração da lactação, isto é, as vacas com maior intervalo de partos responderam por maior produção de leite. Como conseqüência, os animais mais pesados e submetidos à hormonioterapia produziram mais 
leite em razão da maior duração de lactação e pelo fato de terem concebido mais tardiamente.

Segundo Freitas et al. (1997), o período de serviço tem influência sobre a produção de leite na lactação em curso. Esses autores, ao trabalharem com vacas mestiças, cujo período de serviço médio foi de 135 dias, verificaram que ele influenciou a produção total de leite e a produção até 305 dias de lactação.

Tabela 7. Produção de leite por dia de intervalo entre partos $(\mathrm{kg} / \mathrm{dia})$ de vacas primíparas Holandês-Zebu tratadas ou não com implante de norgestomet (média \pm desvio-padrão)

\begin{tabular}{lcc}
\hline Peso & Tratado & Não-tratado \\
\hline Animais leves & $5,46 \pm 2,00(13)$ & $4,95 \pm 1,73(14)$ \\
Animais pesados & $5,46 \pm 1,46(15)$ & $5,50 \pm 1,49(16)$ \\
\hline \multicolumn{2}{l}{ Os números entre parênteses referem-se ao n. }
\end{tabular}

Estes resultados, muito aquém dos encontrados na literatura, justificam-se pelo fato de se ter usado apenas vacas de primeira cria. Sabe-se que as primíparas ainda estão se desenvolvendo durante a primeira lactação, o que pode prejudicar a produção de leite, caso a alimentação não contemple os requisitos de crescimento.

A taxa de gestação não foi influenciada $(\mathrm{P}>0,05)$ pelos tratamentos (Tab. 8). Verificou-se considerável sobreposição de amplitudes de respostas observadas e prováveis.

Não houve influência $(\mathrm{P}>0,05)$ dos tratamentos hormonais sobre a produção de leite (Tab. 9). A produção dos animais do GII foi $171 \mathrm{~kg}$ de leite a menos do que a do GIII. Houve diferença entre tratamentos quanto à duração da lactação $(\mathrm{P}<0,05)$. A dos animais do GII foi 33 dias menor do que a do GIII, isto é, a maior produção de leite foi conseqüência da duração da lactação.

Tabela 8. Taxa de gestação (\%) em razão do número de dias pós-parto em vacas primíparas mestiças Holandês-Zebu, tratadas ou não com implante de norgestomet

\begin{tabular}{lccc}
\hline Grupo experimental & Período pós-parto & $\mathrm{N}$ & $\%$ \\
\hline GI - 390 a 458 kg com norgestomet & $0-90$ dias & $6 / 16$ & 37,50 \\
GI - 390 a 458 kg com norgestomet & $>90$ dias & $7 / 16$ & 43,75 \\
Total & & $13 / 16$ & 81,25 \\
GII - 464 a 562 kg com norgestomet & $0-90$ dias & $8 / 16$ & 50,00 \\
GII - 464 a 562 kg com norgestomet & $>90$ dias & $8 / 16$ & 50,00 \\
Total & & $16 / 16$ & 100,00 \\
GIII - 374 a 461 kg sem norgestomet & $0-90$ dias & $3 / 16$ & 18,75 \\
GIII - 374 a 461 kg sem norgestomet & $>90$ dias & $11 / 16$ & 68,75 \\
Total & & $14 / 16$ & 87,50 \\
GIV - 452 a 545 kg sem norgestomet & $0-90$ dias & $4 / 16$ & 25,00 \\
GIV - 452 a 545 kg sem norgestomet & $>90$ dias & $12 / 16$ & 75,00 \\
Total & & $16 / 16$ & 100,00 \\
\hline
\end{tabular}

Tabela 9. Produção total de leite $(\mathrm{kg})$ e duração da lactação (dias) de vacas primíparas Holandês-Zebu tratadas ou não com implante de norgestomet (média \pm desvio-padrão)

\begin{tabular}{|c|c|c|c|c|}
\hline \multirow{2}{*}{ Resposta } & \multicolumn{2}{|c|}{ Com hormonioterapia } & \multicolumn{2}{|c|}{ Sem hormonioterapia } \\
\hline & Animais leves & Animais pesados & Animais leves & Animais pesados \\
\hline Produção total (kg) & $2.021,75 \pm 780,76(16)$ & $1.950,87 \pm 525,80(15)$ & $2.121,53 \pm 761,57(15)$ & $2.205,63 \pm 721,12(16)$ \\
\hline Duração média (dias) & $298,06 \pm 42,57 \mathrm{a}, \mathrm{b}(16)$ & $275,27 \pm 33,36 \mathrm{~b}(15)$ & $308,13 \pm 36,28 \mathrm{a}(15)$ & $301,88 \pm 39,07 \mathrm{a}, \mathrm{b}(16)$ \\
\hline
\end{tabular}

Os animais que se tornaram gestantes mais cedo no pós-parto apresentaram menor período de serviço, isto é, houve associação entre as duas características. Vasconcelos et al. (1989), ao avaliarem a duração da lactação de vacas com fração de sangue da raça Holandesa variando de $1 / 2$ a $31 / 32$, verificaram média de 288 dias de duração. Freitas et al. (2001), ao compararem a duração da lactação de diversos grupamentos genéticos, observaram que o período de lactação de fêmeas F1 foi 290 dias, valor semelhante ao descrito por Vasconcelos et al. (1989) e ao obtido neste trabalho. 


\section{CONCLUSÕES}

O norgestomet teve efeito positivo sobre o retorno à atividade ovariana cíclica, independentemente do peso corporal. A hormonioterapia antecipou a concepção em vacas mestiças mais pesadas em 30 dias, embora não tenha interferido na produção de leite, na duração da lactação e na taxa de fertilidade. Conseqüentemente, o retorno para o produtor com a utilização do tratamento hormonal pode advir da redução do intervalo de partos, que resultará em maior número de bezerros produzidos durante a vida produtiva da vaca.

\section{AGRADECIMENTOS}

Os autores agradecem a parceria entre a EPAMIG e a Escola de Veterinária que permitiu a execução deste trabalho, e à FAPEMIG, pelo financiamento do projeto.

\section{REFERÊNCIAS BIBLIOGRÁFICAS}

ALMERAYA, A.I.P.; HIDALGO, C.G. Utilización de progestágenos para la manipulación del ciclo estral bovino. Vet. Mex., v.23, p.31-36, 1992.

BARBOSA NETO, A.C.; FACÓ, O.; MARTINS FILHO, R. et al. Desempenho reprodutivo de bovinos leiteiros mestiços criados no litoral do Ceará. In: REUNIÃO ANUAL DA SOCIEDADE BRASILEIRA DE ZOOTECNIA, 38., 2001, João Pessoa, PB. Anais... João Pessoa: SBZ, 2001. p.450-451.

BELLOSO, E.S.; MARTÍNEZ, G.P.; ONDIZ, A.D. et al. Improvement of reproductive performance in crossbred zebu anestrous primiparous cows by treatment with norgestomet implants or $96 \mathrm{~h}$ calf removal. Theriogenology, v.57, p.1503-1510, 2002.

CONOVER, W.J. Practical nonparametric statistics. New York: Wiley, 1980. 493p.

DISKIN, M.G.; MACKEY, D.R.; ROCHE, J.F. et al. Effects of nutrition and metabolic status on circulating hormones and ovarian follicle development in cattle. Anim. Reprod. Sci., v.78, p.345-370, 2003.

DRACKLEY, J.K. Biology of dairy cows during the transition period: the final frontier? J. Dairy Sci., v.82, p.2259-2273, 1999.

DRIANCOURT, M.A. Regulation of ovarian follicular dynamics in farm animals. Implications for manipulation of reproduction. Theriogenology, v.55, p.1211-1239, 2001.

FERREIRA, A.M. Efeito da amamentação e do nivel nutricional na atividade ovariana de vacas mestiças leiteiras. 1990. 134f. Tese (Doutorado em Zootecnia) - Universidade Federal de Viçosa, Viçosa, MG.

FERREIRA, A.M. Manejo reprodutivo e eficiencia da atividade leiteira. Coronel Pacheco: Embrapa - CNPGL, 1991. 47p. (Documentos Embrapa - CNPGL, 46).

FREITAS, A.F.; TEIXEIRA, N.M.; DURÃES, C.M. Período de serviço e sua influência sobre a produção de leite de vacas mestiças europeuzebu. Rev. Bras. Zootec., v.26, p.1103-1168, 1997.

FREITAS, M.S.; DURÃES, M.C.; FREITAS, A.F. et al. Comparação da produção de leite e de gordura e da duração da lactação entre cinco "graus de sangue" originados de cruzamentos entre Holandês e Gir em Minas Gerais. Arq. Bras. Med. Vet. Zootec., v.53, p.708-713, 2001.

GARCIA-WINDER, M.; LEWIS, P.E.; TOWNSEND, E.C. et al. Effects of norgestomet on follicular development in postpartum beef cows. J. Anim. Sci., v.64, p.1099-1109, 1987.

INGVARTSEN, K.L.; BOISCLAIR, Y.R. Leptin and the regulation of food intake, energy homeostasis and immunity with special focus on periparturient ruminants. Dom. Anim. Endocrinol., v.21, p.215-250, 2001.

LARSON, R.L.; KIRACOFE, G.H. Estrus after treatment with syncro-mate $\mathrm{B}$ in ovariectomized heifers is dependent on the injected estradiol valerate? Theriogenology, v.44, p.177-187, 1995.

NETT, T.M. Function of the hypothalamichypophysial axis during the post-partum period in ewes and cows. J. Reprod. Fertil., Suppl., v.34, p.201-213, 1987.

OLIVEIRA FILHO, B.D.; BARNABÉ, M.C.; GAMBARINI, M.L. et al. Emprego de norgestomet associado ao valerato de estradiol (Sincromate B) em vacas apresentando anestro pós-parto. Ars Vet., v.16, p.28-32, 2000. 
SHORT, R.E.; BELLOWS, R.A.; STAIGMILLER, R.B. et al. Phisiological mechanisms controlling anestrus and infertility in postpartum beef cattle. J. Anim. Sci., v.68, p.799-816, 1990.

SINCLAIR, K.D.; MOLLE,G.; REVILLA, R. et al. Ovulation of the first dominant follicle arinsing after day 21 post partum in suckling beef cows. Anim. Sci., v.75, p.115-126, 2002.

SNEDCOR, G.W.; COCHRAN, W.G. Statistical methods. Ames: Iowa State Universty, 1980. $505 \mathrm{p}$.

TROXEL, T.R.; CRUZ, L.C.; OTT, R.S. et al. Norgestomet and gonadotropin-releasing hormone enhance corpus luteum function and fertility of postpartum suckled beef cows. $J$.
Anim. Sci., v.71, p.2579-2585, 1993.

VASCONCELOS, J.L.M.; SILVA, H.M.; PEREIRA, C.S. et al. Aspectos fenotípicos da produção de leite e do período de lactação em vacas leiteiras com diferentes frações de sangue holandês. Arq. Bras. Med. Vet. Zootec., v.41, p.465-475, 1989.

VERNON, R.G.; DENIS, R.G.P.; SØRENSEN, A. Signals of adiposity. Dom. Anim. Endocrinol., v.21, p.197-214, 2001.

WISHART, D.F.; YOUNG, L.M.; DREW, S. B. Fertility of norgestomet treated dairy heifers. Vet. Rec., v.100, p.417-420, 1977.

YAVAS, Y.; WALTON, J.S. Postpartum acyclicity in suckled beef cows: a review. Theriogenology, v.54, p.25-55, 2000. 